\title{
INICIATIVAS DE BRASIL-ESPAÑA PARA GARANTIZAR LA FORMACIÓN Y CONVIVENCIA FAMILIAR DE LAS PERSONAS HOMOSEXUALES A LA LUZ DEL DERECHO CIVIL
}

\author{
INITIATIVES OF BRAZIL-SPAIN TO GUARANTEE THE FORMATION AND FAMILY \\ COEXISTENCE OF HOMOSEXUAL PEOPLE IN THE LIGHT OF CIVIL LAW
}

\section{Douglas Verbicaro Soares}

Universidade Federal de Roraima, Boa Vista, RR, Brasil. E-mail: douglas_verbicaro@yahoo.com.br

\author{
André Lucas Silva Rodrigues
}

Universidade Federal de Roraima, Boa Vista, RR, Brasil. E-mail: andrelukas_silva@hotmail.com

\section{Karen Santos da Silva}

Universidade Federal de Roraima, Boa Vista, RR, Brasil. E-mail: karensantos96188@gmail.com

DOI: https://doi.org/10.46550/ilustracao.v2i2.67

Recebido em: 14.04.2021

Aceito em: 14.07.2021

\begin{abstract}
Resumen: Este estudio tiene como objetivo discutir medidas para la integración de la diversidad sexual en el campo del derecho civil y en la sociedad brasileńa, destacando y presentando las posibles medidas que se pueden implementar con miras a esta integración. En este sentido, tales medidas tienen como objetivo hacer efectivas las garantías y derechos de los grupos sociales vulnerables y lograr la igualdad de trato entre los ciudadanos brasileños. Para la metodología de estudio se utilizó un enfoque teórico cualitativo, basado en la investigación bibliográfica. El estudio destacó importantes y posibles medidas para apuntar a la integración de la diversidad sexual en Brasil, con inspiración el en el modelo español, entre las cuales: cambios en la redacción de los textos normativos de la Constitución y, también, en las normas infra constitucionales, con la inclusión de definiciones y terminologías que abarcan la temática sexual. Como, por ejemplo: la diversidad de género de las personas; facilitar el reconocimiento de la unión estable entre personas del mismo sexo y la conversación de esta para el matrimonio civil; iniciativas públicas centradas en la atención y la prestación de servicios de afrontamiento; elaboración de Planes de Igualdad para casos de desigualdad entre otras medidas. El estudio concluye por la existencia de discriminaciones a las personas homosexuales en Brasil.
\end{abstract}

Palabras clave: Igualdad. Discriminación. Derechos Humanos.

Abstract: This study aims to discuss measures for the integration of sexual diversity in the field of civil law and Brazilian society, highlighting and presenting the possible measures that can be implemented with a view to this integration. In this sense, such measures aim to give effect to the guarantees and rights of vulnerable social groups and to achieve equal treatment among Brazilian citizens. For the study methodology, a qualitative theoretical approach was used, based on bibliographic research. The study highlighted important and possible measures aimed at the integration of sexual diversity in Brazil, with inspiration in the Spanish model, such as: changes in the wording of the normative texts of the Constitution 
and, also, in the infra-constitutional norms, with the inclusion of definitions and terminologies that cover the sexual theme. Such as: the gender diversity of people; facilitate the recognition of and conversation between the stable same-sex union for civil marriage; public initiatives focused on care and the provision of coping services; preparation of Equality Plans for cases of inequality among other measures. The study concludes by the existence of discrimination against homosexual people in Brazil.

Keywords: Equality. Discrimination. Human Rights.

\section{Introducción}

- 1 estudio tiene como objetivo discutir medidas para la integración de la diversidad sujeto a la voluntad momentánea de los gobiernos, especialmente cuando dicho gobierno tiene convicciones conservadoras. Así, el discurso muestra importancia social, ya que la realización de garantías y derechos de los grupos sociales vulnerables es fundamental para el logro de la igualdad de trato entre los ciudadanos brasileños.

Así, ¿el estudio destaca qué medidas se pueden tomar para lograr esta igualdad?

Entre ellas: cambios en la redacción de los textos normativos de la Constitución y en las normas infra constitucionales, incluyendo definiciones y terminologías que abarcan la diversidad sexual y de género de las mujeres. personas; facilitar el reconocimiento de la unión estable entre personas del mismo sexo y la conversación de esta para el matrimonio civil; iniciativas públicas centradas en la atención y la prestación de servicios de afrontamiento; elaboración de Planes de Igualdad para casos de desigualdad; creación de políticas educativas con la implementación de proyectos docentes que permitan cambiar la situación actual de exclusión, entre otras iniciativas.

De acuerdo con la metodología utilizada en el artículo, se utilizó un enfoque teórico cualitativo, basado en la investigación bibliográfica.

Para la estructura del estudio se diseñaron tres apartados. El primero aborda el reciente cambio en la comprensión jurídica de las uniones entre personas del mismo sexo y la dificultad que enfrentan las parejas homosexuales ante las dificultades burocráticas y legales del país. El segundo apartado trataba de las medidas para la integración de la orientación sexual homosexual en el derecho civil español. Finalmente, la tercera parte de la investigación presentó las posibles iniciativas para la integración de la diversidad sexual en la sociedad brasileńa.

\section{La realidad del Derecho civil normalizado en sociedad}

Nos ha parecido viable hacer algunos comentarios sobre las medidas para conciliar la vida de los ciudadanos brasileños en la esfera del Derecho Civil de Brasil. Como punto de partida, añadimos que el lema de la adopción de niños por parejas homosexuales sigue presentado una verdadera burocracia en los trámites procesales, haciendo con que la espera pueda durar muchos años. Hecho eso que generan una compleja incertidumbre en cuanto a la viabilidad de la adopción en el país.

Segundo informaciones presentes en el "Manual de Comunicação LGBT":

El casamiento y la constitución de la familia son derechos negados hasta este período supra citado en la parcela de la población GLBT. En 1995, ha sido 
presentado en el Congreso Nacional el Proyecto de Ley 1.151/95, que oficializaría la unión entre personas del mismo sexo. Proyecto este que nunca ha llegado a pasar por la aprobación y, en 2009, fue sustituido por el Proyecto de Ley 4.914/09 (ABGLT, 2010, p. 34).

Hecho que demuestra poco interés y la fácil sujeción de los temas de los colectivos en situación de vulnerabilidad conforme la voluntad momentánea de los Gobiernos. Si en épocas en que los representantes de los ciudadanos son de ideas más progresistas y menos conservadoras, parece de ser que los lemas entendidos como tabúes siguen un camino más célere para la apreciación. Si, por otro lado, los líderes son de características conservadoras, el camino se presenta más complicado, incluso proyectos son abandonados, paralizados o definitivamente archivados.

En este momento reiteramos que la posibilidad de unión civil ha llegado hace pocos años, es decir fue en el día 05 de mayo de 2011, a partir de una decisión del Supremo Tribunal Federal de Brasil, cuando examinaba una serie de acciones en las cuales se argumentaba que negar el derecho de unión a los homosexuales violaba varios principios de la Constitución Federal, entre ellos, la igualdad y libertad (VERBICARO SOARES, 2012, p. 48).

Con ese fundamento, las parejas homosexuales pueden ser incluidas en el régimen jurídico de la unión estable y beneficiarse de este hecho. Las acciones frente al Superior Tribunal Federal pedían que la unión estable homosexual fuese reconocida por el Ordenamiento Jurídico brasileño como entidad familiar. Con la decisión de la Corte Suprema de Brasil (CASAMENTO CIVIL IGUALITÁRIO, 2014), las personas con orientación homosexual pasan a tener derechos a herencia, inscripciones de la pareja en la seguridad social y en planes de salud (BRASIL, 2011).

En este momento único en la historia de Brasil, las parejas de homosexuales que ya tenían el reconocimiento de las uniones de hecho en las notarías del país, empiezan a solicitar la conversión de las mismas en casamiento civil, pero como todavía ese derecho es muy reciente, las interpretaciones cuanto al tema son contradictorias y en algunos casos ese precepto encuentra obstáculos por la interpretación subjetiva de los jueces de Tribunales Inferiores, que por un motivo u otro, no reconocen esa equiparación, lo que hace con que las personas afectadas por decisiones negativas, que tengan que seguir con los tramites hasta los niveles más altos para tener efectuado sus derechos (CASAMENTO CIVIL IGUALITÁRIO, 2014).

Se hace oportuno citar que este pasó más en el reconocimiento de derechos ha sido iniciado por el Poder Judiciario de Brasil, para satisfacer a las demandas presentadas para regular una situación que ya existía en la sociedad. El derecho en este sentido ha sido llamado para proteger a estos casos y al clamor social existente. En el mismo sentido, por el mundo (CASAMENTO CIVIL IGUALITÁRIO, 2014), otras manifestaciones del Ordenamiento Jurídico de distintos Estados, también, actuaron a favor del reconocimiento del casamiento civil entre parejas del mismo sexo, como por ejemplo lo ocurrido en Estados Unidos: a) la Suprema Corte de Iowa, b) Suprema Corte de Massachussets, c) Suprema Corte de California, d) Suprema Corte de Connecticut.

En África del Sur, la manifestación de la Corte Constitucional también ha sido a favor del reconocimiento del casamiento civil entre personas del mismo sexo. Del mismo modo en que la Corte de Apelación de Ontario, en Canadá.

No podemos dejar de mencionar en este estudio que existe en vigor una propuesta de 
enmienda a la Constitución brasileńa para alteración de esta cuanto al artículo 226:

Art. 226: la familia, base de la sociedad, tiene especial protección del Estado; $\$ 1^{\circ}$

- El casamiento es civil y gratuito su celebración; $\$ 2^{\circ}-$ El casamiento religioso tiene efecto civil, en los términos de la ley; $₫ 3^{\circ}$ - Para efecto de protección del Estado, es reconocida la unión estable entre el hombre y la mujer como entidad familiar, debiendo la ley facilitar su conversión en casamiento (VERBICARO SOARES, 2012, p. 48).

Con el cambio sugerido por el Poder Legislativo, en artículo 226 de la Constitución Federal se quedaría de la siguiente forma:

La familia, base de la sociedad, tiene especial protección del Estado; $₫ 1^{\circ}-\mathrm{El}$ casamiento es civil y gratuito su celebración, será realizado entre dos personas, y en cualquier de los casos, tendrá los mismos requisitos y efectos para los cónyuges del mismo o diferente sexo; $\$ 2^{\circ}-$ El casamiento religioso tiene efecto civil, en los términos de la ley; $₫ 3^{\circ}-$ Para efecto de protección del Estado, es reconocida la unión estable entre dos personas, sea del mismo o diferente sexo como entidad familiar, debiendo la ley facilitar su conversión en casamiento.

De este modo, se cambiaría, principalmente, los términos de la unión estable entre hombre y mujer (VERBICARO SOARES, 2015, p. 282), ampliando su significado para personas de este o diferente sexo (definición integradora de la diversidad humana).

\section{Medidas para la integración de la orientación sexual homosexual en el ámbito del derecho civil español}

No podemos dejar que tratar del caso Español, que ha tenido que alterar preceptos del Código Civil en materias de contraer matrimonio. La apuesta multicultural del sistema jurídico español para atender las exigencias democráticas de igualdad de trato entre los ciudadanos.

Según la Ley 13/2005 y la Resolución-circular de 29 de julio de 2005, de la Dirección General de los Registros y del Notariado, sobre matrimonios civiles entre personas del mismo sexo, que en sus determinaciones expresas informa que: la primera, la Ley 13/2005:

Se ha procedido a una imprescindible adaptación terminológica de los distintos artículos del Código Civil que se refieren o traen causa del matrimonio, así como de una serie de normas del mismo Código que contienen referencias explícitas al sexo de sus integrantes. En primer lugar, las referencias al marido y a la mujer se han sustituido por la mención a los cónyuges o a los consortes. En virtud de la nueva redacción del artículo 44 del Código Civil, la acepción jurídica de cónyuge o de consorte será la de persona casada con otra, con independencia de que ambas sean del mismo o de distinto sexo. Subsiste no obstante la referencia al binomio formado por el marido y la mujer en los artículos 116, 117 y $118 \mathrm{del}$ Código, dado que los supuestos de hecho a que se refieren estos artículos sólo pueden producirse en el caso de matrimonios heterosexuales. Por otra parte, y como resultado de la disposición adicional primera de la presente ley, todas las referencias al matrimonio que se contienen en nuestro ordenamiento jurídico han de entenderse aplicables tanto al matrimonio de dos personas del mismo sexo como al integrado por dos personas de distinto sexo (BOE N. 157/2005).

Hemos hecho en su momento una crítica al modelo español, que la reiteramos en referencia al contenido de la Resolución-circular, es interesante trabajar con la cuestión de los matrimonios entre personas del mismo sexo, pero que de nacionalidades distintas o del mismo origen, es decir: un español y extranjero, o en los casos en que ambos son extranjeros, en sus 
preceptos:

Así resulta de lo dispuesto en el párrafo segundo que se añade al artículo 44 del Código, conforme al cual «El matrimonio tendrá los mismos requisitos y efectos cuando ambos contrayentes sean del mismo o de diferente sexo». Ahora bien, la citada Ley 13/2005 no ha introducido ninguna modificación en las normas del Derecho Internacional Privado español, lo que suscita el interrogante de cuál será la ley aplicable a los matrimonios mixtos de espańol/a y extranjero/a en materia de capacidad matrimonial, en particular por lo que se refiere al posible impedimento de identidad de sexo, o dicho en otros términos, si la permisión de la ley española respecto de los matrimonios integrados por personas del mismo sexo se extiende también en presencia de elementos personales de extranjería, esto es, cuando uno o ambos contrayentes sean de nacionalidad extranjera (BOE N. 188/2005).

Este tema mencionado no se encuentra regulado en el Ordenamiento Jurídico brasileño, lo que la problemática seguirá presente en situaciones análogas a española (VERBICARO SOARES, 2012, p. 50).

Se hacemos referencia a la unión y regulación de esta unión tenemos una crítica al modelo español cuando a la efectividad cuanto las "parejas de hecho". Debido a las especificidades de cada Comunidad Autonómica sobre algunas exigencias para permitir esa protección administrativa y jurídica a parejas del mismo sexo. La Comunidad de Castilla y León suspende en la eficiencia y respeto a lo que determina el propio Ordenamiento Jurídico español, desde el período de 2011 hasta 2014, no hemos detectado cambios en este sentido y el trato a personas que desean regular su situación afectiva tendrán una dificultad añadida.

Los casos de abusos y problemas sobre el tema en la Comunidad de Castilla y León son los más distintos. Resaltamos, en este momento, que el problema mencionado se presenta cuando las parejas intentan regularizar y registrarse en el procedimiento de uniones de hecho en el registro correspondiente en Salamanca. Una vez que reciben, determinadas informaciones, por los funcionarios de la Administración pública, que los trámites para reconocimiento, no solamente entre parejas del mismo sexo, cómo que, de sexo diverso, en permitir que lo hagan entre pareja de español/a y otra persona de nacionalidad de no miembro de la unión europea.

La problemática está en la ausencia, abismo legal en los temas de los visados de estudios para extranjeros, lo que según funcionarios de la administración de Salamanca, recibiendo determinación de la sede de Valladolid, informa que la solicitud de pareja de hecho entre español/a y estudiante con visado de estudios no es permitida, por lo menos en la Comunidad de Castilla y León, una vez que la tarjeta de extranjero con visado de estudios no equivale a la tarjeta de residencia-permiso de residencia en vigor en caso de extranjero no comunitario (BOCYL 21/07/2010) y no tener ese permiso de residencia, impediría el reconocimiento de la unión de hecho entre estas parejas.

Con base en el Reglamento del CYL, en su artículo 4: tópicos para la inscripción en el Registro de Uniones de Hecho. 1. Los miembros de la pareja deberán cumplir los siguientes requisitos a la fecha de presentación de su solicitud de inscripción en el Registro de Uniones de Hecho: convivencia que implique una relación de afectividad entre los solicitantes análoga a la conyugal actual y durante al menos los seis meses inmediatamente anteriores, o durante seis meses continuados; residencia actual en la Comunidad de Castilla y León, ser mayores de edad o menores emancipados; no tener relación de parentesco por consanguinidad o adopción hasta 
el segundo grado; ser su estado civil el de soltero/a o viudo/a o divorciado/a; no formar unión de hecho con otra persona; no estar incapacitado judicialmente (Orden FAM/1597/2008, de 22 de agosto) (VERBICARO SOARES, 2012, p. 50).

Ya en el año de 2012 alertamos sobre las dificultades presentadas y trabajamos con una simple pregunta: ¿Cómo están siendo realizados los trámites para estos casos de los integrantes de las parejas que tienen un permiso de estudios? Reiteramos que la situación no se ha cambiado en 2014 y la alternativa que nos deparamos ha sido la de hacerlo con el empadronamiento de una de las personas de la pareja, previamente empadronada en otras ciudades de Comunidades Autonómicas distintas, que no colocan obstáculos cuanto a esa regulación, como por ejemplo: Comunidades de Extremadura o la de Madrid, que piden el pasaporte de los extranjeros no comunitarios como documentos necesarios para inscripción en el Registro Civil de esas Comunidades (VERBICARO SOARES, 2012, p. 50).

En Brasil, para regular la situación de los registros se puede solicitar el reconocimiento de la pareja de hecho por vía jurídica, que tiene un trámite más largo y costoso, por otro las solicitudes mediante notario, con un valor económico mucho inferior y que es entregue la documentación en el mismo día, siendo más cómoda la realización de la segunda opción, incluso para los casos entre parejas entre nacional (brasileño) con extranjero.

En 2012 hemos tenido la posibilidad de hablar con una pareja homosexual de brasileño con un español en Belém, ellos han optado por realizar en un notario el contrato de unión estable para reconocer su situación en Brasil. Además, con lo certificado, el extranjero (español) podrá solicitar el cambio de su situación legal en el país, es decir, alterar el hecho de ser turista (posibilidad de estar en Brasil por tres meses) para un visado temporario o permanente para la pareja del nacional brasileńo (VERBICARO SOARES, 2012, p. 50).

Este dispositivo que altera la situación y concede derechos a parejas homosexuales con vínculos de unión estable. El dispositivo es la Resolución Normativa 77/2008 del Consejo Nacional de Inmigración, de 11 de febrero de 2008. Según sus preceptos: Art. 1 Las solicitaciones de visado temporario o permanente, o de autorización de permanencia para compañero o compañera, en unión estable, sin distinción de sexo, deberán ser examinadas al amparo de la Resolución supra citada, relativa a las situaciones especiales o casos omisos, y de la Resolución Normativa 36/1999, sobre reunión familiar (VERBICARO SOARES, 2012, p. 51).

El reconocimiento de los hechos será acreditado de acuerdo con los preceptos del artículo 2 de la Resolución 77/2008: La comprobación de la unión estable podrá ser hecha por uno de los siguientes documentos: I - atestado de unión estable emitido por órgano del Gobierno del país de procedencia del llamado; II - comprobación de unión estable emitida por juzgado competente en Brasil u autoridad correspondiente en el exterior (ABGLT.ORG, 2008).

Los cambios normativos de España sirven como ejemplo al que podrá sucederse en Brasil, cuanto a las futuras modificaciones de la legislación brasileña después de la Decisión inédita del Supremo Tribunal Federal en reconocer la posibilidad del casamiento entre personas del mismo sexo, sumadas al proyecto de emenda constitucional del exdiputado Federal Jean Wyllys (VERBICARO SOARES, 2015, p. 288) que objetiva, entre otros temas, las sustituciones de determinados preceptos (definiciones) de las normas de Brasil, para conseguir la igualdad de trato de modo más amplio, para toda la sociedad, sin discriminación. 


\section{Las iniciativas para la integración de la diversidad sexual dentro de la sociedad brasileña}

Bajo esas ideas, resolvemos optar por hacer algunas consideraciones sobre ciertos instrumentos creados, en la sociedad brasileña, en los últimos años, cuanto a la promoción de la igualdad de oportunidades entre los ciudadanos. Por esta razón, se hace importante enfocar en el ámbito del Estado, iniciativas que enfoquen en el cuidado y en los servicios públicos necesarios para enfrentarlos, es decir, la organización social de cuidar parece ser según este criterio, una de las llaves para alcanzar una mayor equidad en Brasil (TORNS, 2008, p. 71).

Dicha Igualdad se basada en el fomento de los idearios democráticos igualitarios y en políticas a favor de la incorporación de los grupos históricos socialmente excluidos de participación a la vida social, sin cualquier obstáculo en dos diversos segmentos de la sociedad brasileña, sea en la vida social, económica, laboral, cultural, política, sexual, etc., visibilizando si fuera el caso, la implementación de acciones positivas para facilitar esa igualdad deseada.

Corroborando con esta idea, citamos a León Alonso, que nos aclara que:

la igualdad de oportunidades entre hombres y mujeres ha de estar presente en las políticas y acciones públicas. La igualdad se convierte en una especia de principio-guía, un faro que deberá orientar todas las actuaciones de los poderes del Estado. Esta técnica de articulación de las políticas de igualdad se designa con la expresión inglesa gender mainstreaming, que en España hemos traducido como transversalidad del género (LEÓN ALONSO, 2014, p. 18-9).

Añadimos esa idea, de un modo análogo a las políticas implementadas en España, también creadas para generar una igualdad de trato entre los grupos vulnerables, en este caso, a las personas homosexuales, donde se designará por parte del Estado, políticas de acción para lograr la igualdad de trato de esas personas en relación con los demás ciudadanos.

González Bustos nos aclara que en correspondencia a las materias sobre medidas de incentivo para promover la igualdad de oportunidades estarían: todo el conjunto de medidas establecidas en los textos legales y que inciden sobre la igualdad (GONZÁLES BUSTOS, 2010, p. 239). Igualdad para un efectivo trato entre hombres y mujeres. Para nuestro estudio, también ampliamos esa idea para las personas homosexuales, en este caso, hombres y mujeres que se encuentran en situación de desigualdad frente a otros hombres y a mujeres (heterosexuales en su mayoría), donde las personas con orientación sexual se deparan con mayor dificultad para superar las situaciones de vulnerabilidad en Brasil, sean en el ámbito social, económico, laboral, político, etc. También llamamos la atención para las mujeres lesbianas, que acaban sufriendo un doble problema, es decir: sufren discriminación tanto por ser mujeres y lesbianas.

En ese sentido, debemos buscar instrumentos para posibilitar el trato igualitario, no solamente cuando tratamos de los temas de género, como también los de identidad de género. De ese modo es importante que, entre las alternativas presentadas por los Estados, en nuestro caso el modelo de Brasil, en la creación de Planes de Igualdad para los casos de desigualdad, principalmente, los que visibilizan los colectivos en situación de exclusión social, como son los homosexuales.

En ese sentido citamos a Alexy en la justificación viable de la utilización de las políticas afirmativas pala la integración de personas en situación de desigualdad frente a los demás ciudadanos. Para esas personas y la aplicación del principio de la igualdad se aplicaría en el trato igual a los iguales y desigual a los desiguales (ALEXY, 2007, p. 215), donde la explicación por 
utilizar medidas de acciones positivas está en esa situación vislumbrada de desigualdad. Para que se pueda cambiar la realidad de eses desniveles, se aplican eses instrumentos hasta que los desiguales puedan ocupar patatares de igualdad y superación de la vulnerabilidad.

Para dilucidar nuestros argumentos usaremos a seguir, de modo análogo, las ponderaciones de Souto Galván que, al referirse sobre la viabilidad de los planes de igualdad en la perspectiva de la igualdad entre hombre y mujeres, trabaja con ciertas ideas que nos servirán para basar nuestra fundamentación. La autora trató en su obra algunos puntos que merecen destaque, como ha sido el caso de: la introducción de la perspectiva de género en las políticas públicas, igualar la vida económica de las mujeres y hombres, participación en la tomada de decisiones, promoción de la cualidad de vida, fomento de la igualdad civil, transmisión de valores y actitudes igualitaria y la cooperación (SOUTO GALVÁN, 2012, p. 136-7).

Defenderemos la necesidad de: a) implementación de la vertiente de los lemas de diversidad sexual en las políticas públicas de Brasil, así como también, motivar el sector privado en seguir en ese mismo camino, buscando la inclusión de las personas homosexuales en sociedad. B) Para los casos de salarios de las mujeres y mujeres lesbianas, resaltamos la viabilidad de permitir la incorporación igualitaria de las mujeres en el ámbito laborar, con igualdad de remuneración hacia a sus compañeros hombres, principalmente en el ámbito militar; c) Del mismo modo que todos los planes, estrategias, políticas deberán ser pensadas en permitir que las mujeres, las mujeres lesbianas y a los gais puedan incorporarse en los más diversos sectores, como por ejemplo que en las FF.AA., de Brasil, puedan ocupar cargos de dirección, comando y ejercer también según el modelo jerárquico militar el liderazgo, independiente de su sexo u orientación sexual; d) Se hace viable incentivas la calidad de vida y el reconocimiento de ese modo de la dignidad de las personas homosexuales, permitir que esas personas tengas igual trato a los demás, es decir: que puedan tener los mismos derechos, ingreso a un sistema de salud digno, una educación sin discriminación y basada en la trasmisión de los valores igualitarios entre todas las personas, con releva a lo tema de la diversidad sexual e etc.; e) Incentivar la inclusión social de las personas homosexuales en la sociedad brasileña, acciones conjuntas entre las ONGs y la sociedad civil en la promoción de la participación ciudadana y la integración de los colectivos que sufren exclusión; f) Fomentar la cooperación del Estado brasileño con el plano internacional, tanto en la esfera nacionalizada como internacional, por medio de experiencias satisfactorias y de éxito en otros países que puedan ser empleadas y adaptadas a la realidad de Brasil.

Los hechos comentados nos hacen reflexionar sobre unos comentarios de Preciado, que al citar otra importante feminista, el libro clásico de Mary Wollstonecraft, "A vindication of the rights of woman":

Publicado en 1792, tenía varias reivindicaciones distintas expuestas en el programa general de "defensa" que ella delineó. Los derechos a los que se refería incluían no solamente algunos particularmente relacionados con el bienestar de la mujer, sino también derechos relacionados con la libre condición de agente de la mujer (SEN, 2002, p. 220-1).

Son los deseos democráticos de libertad individual de cada ciudadano en tener un control sobre los roles impositivos sociales, culturales e históricos en influenciar la vida de las personas, determinado que, en ese caso, las mujeres puedan emanciparse de una situación de sumisión creada y que la victime en un cuadro jerarquizado inferior en relación con los hombres. Los clamores de libertad sirven como inspiración para que las mujeres reivindiquen y consigan 
derechos hasta entonces negados. Son los cambios de una no participación social hacia a la integración y lucha por la incorporación igualitaria, bajo el lema de igual trato, iguales derechos entre hombres y mujeres.

Para Elmôr, los estudios desarrollados, a partir de la década de 20, representan la esencia sobre la discusión de la masculinidad, pues será cuando se empiezan a tratar de los temas del masculino y del femenino de un modo más científico. Se enfatiza que en la década de los 60, junto con los movimientos feministas y de los movimientos de los homosexuales, se amplían los estudios sobre la masculinidad y feminidad y los nuevos rumbos de estos términos, principalmente en Estados Unidos e Inglaterra (GERMANO ELMÔR, 2002, p. 33).

Corroboraremos la formación de nuestro trabajo con las ideas de Gil Rodríguez, que se manifiesta sobre la importancia del feminismo hacia a la lucha por la igualdad de trato entre hombres y mujeres de un modo significativo. Para la autora: no fue hasta 1964 que se definió la expresión género, y fue porque un psiquiatra, Roberto Stoller, buscaba una palabra para poder diagnosticar aquellas personas que, aunque poseían un cuerpo de hombre, se sentían mujeres (GIL RODRÍGUEZ, 2007, p. 14). El argumento del determinismo biológico, que restringía la comprensión sobre la naturaleza humana y que supuestamente limitaba a una persona a su sexo biológico e inmutable, ha tenido en esta esencia la motivación para la incidencia de la discriminación sobre las mujeres, por considerarlas como inferiores a los hombres, debido a la propia naturaleza. De este modo, el movimiento feminista luchando junto contra ese posicionamiento, se posiciona con un cambio de mentalidad, en el medio social, del ambiente donde se viva y se incida padrones culturales.

Se puede aducir que según la autora supra citada: que los estudios al procuraren definir, de modo amplio, en las esferas del público y del privado, su propio espacio en la política, en la economía y en las cuestiones relacionadas con la sexualidad, tanto hombres como mujeres se organizaron para luchar contra la discriminación que sufrían, proponiendo otras mentalidades, distintos comportamientos, palabras y perspectivas para las relaciones entre los sexos, cuestionado de este modo el modelos de masculinidad hegemónico mayoritario en sociedad occidental, es decir: un modelo creado por blancos, heterosexuales y dominantes (GIL RODRÍGUEZ, 2007, p. 14).

En la misma línea de raciocinio Duarte Vilar resalta que:

En las últimas décadas, asistimos a profundos cambios en el modo de cómo se habla y se vive la sexualidad en la sociedad occidental. Cambios estos que tienen una expresión en el plano macrosocial, en la emergencia de actitudes más liberales sobre la sexualidad, que impregnan los diferentes discursos - dos técnicos, de las leyes y de las políticas y del propio senso común. En el plan de las vivencias individuales, estos cambios se expresan en nuevas formas de pensar, sentir, actuar en los dominios de la vida y del comportamiento sexual (VILAR, 2010, p. 2012).

Sin duda alguna debemos reconocer y estamos de acuerdo con el autor que cada vez más la proyección sobre el tema de la orientación sexual homosexual en un modo global se ha dado a conocer.

Se por medio de los medios de comunicación, películas, seriados o por medio de personajes públicos que han dado a conocer sus orientaciones sexuales. Como ejemplos podemos citar seriados de los Estados Unidos o Ingleses, de gran audiencia internacional que 
han trabajado sobre la materia y han ayudado a la sociedad internacional a familiarizarse con la homosexualidad: Brokeback Mountain, Una casa en el fin del mundo, Six Feet Under, Queer as Folk, Grey's Anathomy, Modern Family, Brothers \& Sisters, Dowton Abbey, Looking, L-World, True Blood, Melrose Place y muchos otros. Personajes en el área artística también han destacado su homosexualidad o bisexualidad y contribuyeron para una discusión social sobre ella, como han sido: George Michael, Ricky Martin, Tizziano Ferro, Adriana Calcanhoto, Marina Lima, Ana Carolina, Caetano Veloso, etc.

Conforme adentramos sobre el tema de la sexualidad, notamos que la sociedad occidental pasó de un modelo sexual básicamente reproductivo, donde las prácticas sexuales servían o eran entendidas como un medio para la perpetuación de la especie humana, blindada por un modelo heterosexual dominante y marcha hacia a un modelo cambiante de valoración del estado de placer entre los individuos en la satisfacción de sus necesidades sexuales, es decir: se abre paso a la idea mucho más amplia sobre la sexualidad humana y a la satisfacción de la intimidad de cada individuo y la afectividad personal que escoja vivir en sociedad.

Vilar nos recuerda que ha sido en las últimas décadas del siglo XX, más específicamente en los años sesenta, se expanden las asociaciones de sexología clínica, en la proliferación de programas de formación de terapeutas sexuales y en la oferta de servicios de sexología. En la década de los ochenta, este nuevo movimiento sexual ha influenciado la Organización Mundial de la Salud en la construcción de un nuevo concepto: la salud sexual, donde se buscaría integrar en las políticas, prácticas y servicios de salud esta área hasta entonces ignorada (VILAR, 2010, p. 203).

Por esta razón, los movimientos feministas y los de la comunidad LGBT empujan a la sociedad en los nuevos rumbos sobre la visibilidad social de los lemas de la sexualidad y de reconocimiento de derechos y la igualdad de trato entre todos los ciudadanos, combatiendo, de este modo, las discriminaciones hacia a la diversidad, sea por motivos de sexo, raza, orientación sexual, etc.

Con la misma línea de interpretación se encuentran los argumentos de Alícia Muñoz que al mencionar el caso análogo de la situación en España, haciéndose eco de las demandas del feminismo, y respondiendo a las recomendaciones y compromisos internacionales, ha desarrollado diversas políticas de igualdad (VERBICARO SOARES, 2012, p. 39).

De acuerdo con ese seguimiento, la autora desarrolla sus aportaciones relacionando con uno de los ocho Objetivos del Milenio de las Naciones Unidas - Eliminar la disparidad de género (educación primaria y secundaria, preferentemente para 2005 y, en todos los niveles educativos en el año de 2015. Citase, más específicamente el Marco de Dakar - Senegal 2000, con el objetivo de lograr una educación para todos (VERBICARO SOARES, 2012, p. 39-40).

En ese sentido se manifiesta la Amnistía Internacional: Educación para la Ciudadanía y los Derechos Humanos en Espańa: Un paso correcto, un desarrollo insuficiente. 2008. ¿Lo que representa esta problemática? Se los objetivos no son cumplidos: peligrar gran parte de los avances conseguidos por el movimiento feminista hacia una ciudadanía más inclusiva (VERBICARO SOARES, 2012, p. 40). La igualdad de trato entre hombres y mujeres, así como la inclusión de grupos excluidos se torna más difícil, por esta razón, las políticas de educación son importantes para cambios de realidades, no solamente en el ámbito de España, como nos presentó Amnistía Internacional, pero también un fin a ser implementado en Brasil. 
Cuanto, a esa temática, recordaremos que el Plan Nacional de Políticas para las Mujeres incluye explícitamente que la educación es un instrumento eficaz para combatir las desigualdades entre hombres y mujeres, haciendo referencia clara a los casos de discriminación por orientación sexual como un problema para el Gobierno de Brasil y para la sociedad. De este modo presenta la educación como medio para la reducción de las desigualdades en el ámbito de la enseñanza del país y su posterior reflejo positivo a largo plazo en sociedad (BRASIL, 2013, p. 23-4).

De este modo hacemos la indagación ¿Por qué la educación se presentaría como una solución a los problemas enfrentados en Brasil? La respuesta está en la posibilidad de implementar proyectos de enseñanza que permitan cambiar la situación actual de exclusión ciudadana en la sociedad brasileńa, de modo que valores de igualdad de trato puedan ser seguidos y efectivarlos para la protección de derechos para los brasileños.

Desde el principio del estudio presentado (2008), hemos optado por defender la educación con mecanismo eficaz para combatir las desigualdades sociales en Brasil, principalmente cuanto al tema de la discriminación a las personas en situación de mayor vulnerabilidad (VERBICARO SOARES, 2011, p. 187-8). Por estos hechos se suman nuestra visión sobre algunos de los pilares que consideramos importantes a la hora de entender la importancia sobre el problema enfrentado.

Reconocemos que los problemas son todavía desafiantes. Según el Informe disponible en el mes de julio de 2015, del Fondo de las Naciones Unidas para la Infancia, se ha reducido en 64\% la evasión escolar de niños y adolescentes en la enseñada secundaria, pasando de 19,6\% de alumnos matriculados en 1990, para 7\% en 2013. En el estudio, se presentaban índices de casi 700 mil niños (4-5 años) que estaban fuera de las escuelas. Por otro lado, se visibilizó un incremento en el número de matrículas de adolescentes de 15-17 ańos (EBC, 2015).

En este sentido que se manifiesta el artículo 26 de la Declaración Universal de los Derechos Humanos (VERBICARO SOARES, 2011, p. 187-8), que dispone la visión de que todas las personas tienen derecho a la educación:

Toda persona tiene derecho a la educación. La educación debe ser gratuita, al menos en lo concerniente a la instrucción elemental y fundamental. La instrucción elemental será obligatoria. La instrucción técnica y profesional habrá de ser generalizada; el acceso a los estudios superiores será igual para todos, en función de los méritos respectivos. La educación tendrá por objeto el pleno desarrollo de la personalidad humana y el fortalecimiento del respeto a los derechos humanos y a las libertades fundamentales; favorecerá la comprensión, la tolerancia y la amistad entre todas las naciones y todos los grupos étnicos o religiosos; y promoverá el desarrollo de las actividades de las Naciones Unidas para el mantenimiento de la paz (ARCHIVES OF THE INTERNATIONAL COUNCIL ON HUMAN RIGHTS POLICY, 2014).

Por estos motivos, la educación es presentada como solución de los problemas actuales relacionados con la temática de la diversidad, por ejemplo:

Ayuntamiento lanza campaña por la diversidad en escuelas municipales: [...] Aceptar es una opción, respetar es un deber. [...] La intención de los organizadores es de implementar en las escuelas una pedagogía de la diversidad, en la cual los estudiantes perciban que hay personas diferentes que precisan ser respetadas, sean negros, amarillos o discapacitados. [...] Ningún profesor podrá ser omiso o ajeno delante de un alumno que esté siendo discriminado por ser homosexual o diferente de los otros. [...]Toda lucha por los derechos sexuales acontece en familia, que todavía expulsa, en variados casos, de casa el hijo/a que se revela 
homosexual.

Este texto se relaciona con una experiencia novedosa y didáctica tomada por un miembro del Gobierno, en la época, de una ciudad brasileña, para permitir la inclusión social de las personas, respetando las diferencias de cada individuo en la sociedad (VERBICARO SOARES, 2012, p. 54-5).

Luker complementará esa idea hablando de la formación de dos grupos antagónicos que preceptúan sobre la sexualidad y la enseñanza a ella atribuida. En este sentido, la autora norteamericana comenta que, entre los grupos mencionado, el primer perteneciente a los conservadores del lema de la sexualidad y los segundos denominados de liberares de la sexualidad. Los enfrentamientos en los últimos 40 años sobre ese tema han evidenciado cambios en los temas del sexo, de la familia y de género (LUKER, 2006, p. 151-2).

El primero grupo defiende la manutención de un modelo que viene de un pasado conservador, mientras que los liberares aceptan el presente y esperan crear un futuro mejor. Las discusiones de la manutención de un pasado ideario, mejor, sin alteraciones de lo que ya viene siendo establecido a lo largo de los tiempos, sin afrontar los problemas que surgen normalmente en la propia evolución social de la humanidad. Los liberales se centran en la expectación, de creer que enfrentando la problemática que se interpone en la vida social, los cambios y los aprendizajes auxiliarían en la construcción de un modelo mejor de convivencia y que el futuro traería mejores augurios para la sexualidad y la aceptación de la diversidad (LUKER, 2006, p. 152).

Nos posicionamos en este estudio en relación con las ideas defendidas por el segundo grupo basadas en el discurso de que es necesaria la comprensión social de que la formación de la sexualidad humana para generar la participación ciudadana de reconocimiento de derechos y deberes de los ciudadanos y de implementar la cooperación de estos en el ambiente en que viven. Ese paso se hace indispensable para la adecuación del tema a las realidades más actuales que se generan en los espacios democráticos para la consolidación de un modelo igualitario de trato entre todos.

\section{Consideraciones finales}

Frente a lo que se abordó, es incuestionable la necesidad de modificar las leyes en el ámbito del derecho civil para asegurar a las personas y/o parejas homoafectivos una convivencia social de respeto e igualdad. Para que eso ocurra es importante crear medidas que busquen efectivizar la vivencia digna de esos individuos, que incluso ya fueron citadas en el trabajo. Este proceso de cambio tiene que ocurrir tanto en la conciencia social como por vías legales.

Uno de los puntos iniciales es entender el impacto negativo que afecta la vida de los homosexuales por la falta de reconocimiento de la sociedad con respecto al derecho de libertad de elección a su orientación sexual o identidad de género que cada ser humano posee. Y para una mejor comprensión es fundamental citar uno de los principales principios del derecho, que sirve como norte al sistema jurídico, el Principio de la Dignidad de la Persona Humana que busca garantizar el mínimo existencial de tratamiento que una persona necesita para vivir en sociedad.

La dignidad de la persona humana no solo garantiza el derecho a la educación, al trabajo, a la salud, sino que también consiste en salvaguardar el pleno desarrollo de la personalidad del individuo, sin intervenciones que prohíban las actuaciones de la autodeterminación, 
vetando acción que genere humillación al ser humano y en especial establece los derechos fundamentales. Como bien se sabe, uno de los derechos básicos de cualquier individuo es la formación familiar, que lamentablemente a veces se les niega a las parejas homo afectivas. Sin embargo, para que ese impasse sea superado la realización de medidas es indispensable, como las que ya fueron ejecutadas, el reconocimiento de la unión estable de personas del mismo sexo por el STF (Supremo Tribunal Federal), que permitió a la población LGBT a formalizar sus uniones, y consecuentemente llevó al Consejo Nacional de Justicia (CNJ) a reglamentar la Resolución 175 , con el propósito de impedir que hubiera el rechazo del servicio registral en realizar el matrimonio o conversión de la unión estable de personas homo afectivas en matrimonio.

La adopción también forma parte del derecho de planificación familiar por parejas del mismo sexo que a pesar de los avances aún no ha sido regulado, dificultando a veces la realización de ese proceso, pero el hecho es que esa forma de adopción es una realidad cada vez más presente en la sociedad. Porque incluso con la Ley $\mathrm{n}^{\circ} 12.010 / 09$, al haber incluido varias modificaciones en el Estatuto del Niño y del Adolescente (ECA), no insertó ese modelo de adopción. Aunque la ausencia legislativa permanece, ya se tienen diversas decisiones posibilitando solicitudes de adopción por parejas homosexuales. Permitir estas adopciones respeta la isonomía entre los seres humanos, ya que las personas tienen el derecho de construir su familia de la forma como desean y aún contribuyen a que nińos y adolescentes formen parte de un ambiente familiar estructurado y con afecto.

Otras medidas que también fueron expuestas son iniciativas públicas y privadas que ayuden en el reconocimiento a la diversidad y la representatividad de la orientación sexual, identidad de género y de la familia homo afectiva. Pues en la actualidad, aunque genere polémicas, es más frecuente encontrar comerciales, películas, series, programas de TVs, dibujos que presenten homosexuales. Incluso el propio poder público debería organizar políticas públicas que informe a la colectividad de la importancia de respetar a los diversos grupos sociales, además de programas que incentiven a las empresas a contratar homosexuales, porque es un asunto personal visto como un impedimento para la contratación. Acciones como estas son importantes porque resaltan y colocan como referencias las personas LGBTs, contribuyendo así para la reducción del prejuicio y discriminación en el país. Tener figuras públicas o incluso personas comunes más que ocupen espacios no despreciados en la sociedad genera y fortalece el sentimiento de orgullo de lo que se es, además de ser una herramienta de combate a la homofobia. La representatividad detrás de la percepción de pertenecer a determinado lugar, haciendo con que las personas entiendan que la nueva formación familiar hace parte de la sociedad y ha garantizado los mismos derechos de cualquier ciudadano.

Las iniciativas que implican educación son buenas herramientas para explicar a los niños, adolescentes y adultos que la sociedad es diversa y que, por eso, nuevas formas de autoidentificarse, de interactuar, de constitución familiar van a surgir. Y cuando esto sucede no puede ser visto como diferente y malo, siendo rechazado, es necesario incluir y ampliar los derechos, adecuar nuestras formas de vivir, cambiar pensamientos y opiniones que son tradicionalmente impuestos La educación muestra una nueva forma de comprender y respetar los cambios sociales, pues a partir del momento en que una persona tiene conciencia de eso, ella reproduce y comparte con el ambiente social que frecuenta, haciendo con que otros individuos entiendan, posibilitando una convivencia armoniosa 
De esa forma, aún existen muchos obstáculos a ser superados para que las parejas homo afectivos gocen plenamente de la garantía constitucional de formar una familia, es posible percibir que sus influencias están cada día más presentes en la sociedad.

\section{Referencias}

ABGLT. Manual de Comunicação LGBT. Lésbicas, Gays, Bissexuais, Travestis e Transexuais. Brasilia, Brasil, 2010.

ABGLT.ORG. Resolução Normativa CNI no 77, de 29 de Janeiro de 2008. Disponible en: <http://www.abglt.org.br/port/resnorm77_290108.html>. Acceso en: 30 de mayo de 2021.

ALEXY, Robert. Derechos sociales y ponderación. Fundación Coloquio Jurídico Europeo. Madrid, España, 2007.

ARCHIVES OF THE INTERNATIONAL COUNCIL ON HUMAN RIGHTS POLICY. Artículo 26 de la Declaración Universal de Derechos Humanos. Disponible en: <http://www. ichrp.org/es/articulo_26_dudh>. Acceso en: 31 de mayo de 2021.

BOCYL 21/07/2010.

BOE N. 157/2005.

BOE N. 188/2005.

BRASIL. Plano Nacional de Politicas para as Mulheres 2013-15. Secretaria de Políticas para as Mulheres. Brasília, Brasil: Ed. Movimento, 2013.

BRASIL. Por unanimidade, Supremo reconhece legalidade da união homossexual estável. Disponible en: <http://www.brasil.gov.br/noticias/arquivos/2011/05/05/por-unanimidadesupremo-reconhece-legalidade-da-uniao-homossexual-estavel>. Acceso en: 30 de mayo de 2021.

CASAMENTO CIVIL IGUALITÁRIO. Jurisprudencia. Disponible en: <http:// casamentociviligualitario.com.br/jurisprudencia/>. Acceso en: 30 de mayo de 2021.

DIAS, Maria Berenice. A Família Homoafetiva. Revista Bagoas - UFRN, n. 03, p. 3-7, 2009.

EBC. Brasil reduziu evasão escolar em 64\% com o ECA, diz UNICEF. Disponible en: <http:// agenciabrasil.ebc.com.br/direitos-humanos/noticia/2015-07/brasil-reduziu-evasao-escolar-em64-com-o-eca-diz-unicef>. Acceso en: 31 de mayo de 2021.

GUERIN, Camila Rocha. Adoção e União homoafetiva. Disponible en: <https://ibdfam.org. br/artigos/524/Ado\%C3\%A7\%C3\%A3o+e+uni\%C3\%A3o+homoafetiva. Acesso en: 10 jun. 2021.

GIL RODRÍGUEZ, Eva. La violência de género. Editorial UOC. Barcelona, España, 2007.

GONZÁLES BUSTOS, Ma Ángeles. "La Transcendencia del Sistema de Función Pública en el Desarrollo de la Igualdad de Oportunidades". In: VV.AA.: Formación y objeto del Derecho antidiscriminatorio de género: perspectiva sistemática de la igualdad desde el Derecho público. Ed. Atelier. Barcelona, Espańa, 2010. 
GERMANO ELMÔR, Marilena. Masculinidade em Jogo: um estudo sobre papéis masculinos num grupo adolescente no futebol. Universidad Estadual de Campinas. Facultad de Educación. Disertación de Máster. Campinas, Brasil, 2002.

LEÓN ALONSO, Marta. “45 Respuestas desde el Derecho Constitucional”. In: VV. AA. Violencia de Género e Igualdad en el ámbito rural. Santiago de Compostela, Espańa: Editora Andavira, 2014.

LUKER, Kristin. When sex goes to school: warring views on sex - and sex education - since the sixties. $1^{\circ}$ Edition. W.W. Norton \& Company. New York, NY. United States, 2006.

RIBEIRO, Raiane Celcia. A Adoção de Crianças por Casais Homoafetivos. São Paulo, set 2019. Disponible en: <https://ambitojuridico.com.br/cadernos/direito-de-familia/a-adocao-decriancas-por-casais-homoafetivos/>. Acceso en: 10 jun. 2021.

CNBSP. Colégio Notarial do Brasil. Disponible en: <https://www.cnbsp.org.br/?url_ amigavel=1\&url_source=noticias\&id_noticia=20221\&lj=683>. Acceso en: 10 jun. 2021 .

SEN, Amartya. Desenvolvimento como liberdade. Prêmio Nobel Companhia das Letras. São Paulo, Brasil, 2002.

SOUTO GALVÁN, Clara. Principio de igualdad y transversalidad de género. Madrid, España: Editora Dykinson, 2012.

TORNS, Teresa. El trabajo y el cuidado: cuestiones teórico-metodológicas desde la perspectiva de género. Empírea. Revista de Metodología de Ciencias Sociales. no 15, enero-junio, España, 2008.

VILAR, Duarte. "Desempenho sexual - satisfação e problemas". In: Moura Ferreira, P. Sexualidades em Portugal. Lisboa, Portugal: Ed. Bizâncio, 2010.

VERBICARO SOARES, Douglas. Homossexualidade e Forças Armadas. A busca pela efetividade dos Direitos Humanos no Brasil. Trabajo de Grado. Universidad de Salamanca: Programa de Doctorado Pasado y presente de los derechos humanos, Salamanca, España, 2011.

VERBICARO SOARES, Douglas. La libertad sexual en la sociedad: especial referencia a la homosexualidad en las Fuerzas Armadas Brasileñas. Salamanca, España. Tesis Doctoral. Universidad de Salamanca - USAL: Programa de Doctorado Pasado y Presente de los Derechos Humanos, 2015.

VERBICARO SOARES, Douglas. Las mujeres y las personas homosexuales en las Fuerzas Armadas. Especial referencia a las FFAA brasileñas. Trabajo fin de curso Máster Oficial en Estudios Interdisciplinares de Género. Universidad de Salamanca. Salamanca, España, 2012. 